Published in final edited form as:

Proc SPIE Int Soc Opt Eng. 2019 February ; 10861: . doi:10.1117/12.2514657.

\title{
Reactive oxygen species explicit dosimetry to predict tumor growth for BPD-mediated vascular photodynamic therapy
}

\author{
Tianqi Sheng ${ }^{1}$, Yi Hong Ong ${ }^{1}$, Theresa M. Busch ${ }^{1}$, and Timothy C. Zhu ${ }^{1}$ \\ ${ }^{1}$ Department of Radiation Oncology, University of Pennsylvania, Philadelphia, PA 19104, United \\ States
}

\begin{abstract}
Photodynamic therapy (PDT) is a well-established treatment modality for cancer and other malignant diseases; however, quantities such as light fluence, and PDT dose do not fully account for all of the dynamic interactions between the key components involved. In particular, fluence rate $(\phi)$ effects are not accounted for, which has a large effect on the oxygen consumption rate. In this preclinical study, reacted reactive oxygen species $\left([\mathrm{ROS}]_{\mathrm{rx}}\right.$ ) was investigated as a dosimetric quantity for PDT outcome. We studied the ability of $[\mathrm{ROS}]_{\mathrm{rx}}$ to predict the cure index $(\mathrm{CI})$ after PDT of murine tumors; $\mathrm{CI}=1-\mathrm{k} / \mathrm{k}_{\mathrm{ctr}}$, where $\mathrm{k}$ and $\mathrm{k}_{\mathrm{ctr}}$ are the growth rate of PDT-treated and control(untreated) tumor, respectively. Mice bearing radiation induced fibrosarcoma (RIF) tumors were treated with BPD-mediated PDT at different in-air fluences (22.5, 40, 45, 50, 70 and 100 $\left.\mathrm{J} / \mathrm{cm}^{2}\right)$ and in-air $\phi\left(75\right.$ and $\left.150 \mathrm{~mW} / \mathrm{cm}^{2}\right)$ with a BPD dose of $1 \mathrm{mg} / \mathrm{kg}$ and a drug-light interval of 15 mins. Treatment was delivered with a collimated laser beam of $1 \mathrm{~cm}$ diameter at $690 \mathrm{~nm}$. Explicit dosimetry of initial tissue oxygen concentration, tissue optical properties, and BPD concentration was used to calculate $\left[{ }^{1} \mathrm{O}_{2}\right]_{\mathrm{rx}}$. $\phi$ was calculated for the treatment volume based on Monte-Carlo simulations and measured tissue optical properties. CI was used as an endpoint for four dose metrics: light fluence, PDT dose, and $[\mathrm{ROS}]_{\mathrm{rx}}$. PDT dose was defined as the product of the time-integral of photosensitizer concentration and $\phi$ at a $3 \mathrm{~mm}$ tumor depth. Preliminary studies show that $[\mathrm{ROS}]_{\mathrm{rx}}$ best correlates with $\mathrm{CI}$ and is an effective dosimetric quantity that can predict treatment outcome. The threshold dose for [ROS $]_{\mathrm{rx}}$ is determined to be $0.23 \mathrm{mM}$ and is about 4.3 times smaller than the corresponding value for conventional BPD-mediated PDT using DLI of 3 hrs.
\end{abstract}

\section{Keywords}

photodynamic therapy; explicit dosimetry; macroscopic singlet oxygen model; BPD-mediated PDT; reactive oxygen species

\section{INTRODUCTION}

Photodynamic therapy (PDT) is a used for treatment of cancer and other localized diseases. PDT leads to fewer side effects than radiation and chemotherapy, because it does not involve ionizing radiation and can be well-localized ${ }^{1,2}$. Widespread use of PDT has been stilted due to the difficulty in accurately quantifying the dose. PDT is not only "dynamic" but also multifaceted $^{3,4}$. It incorporates light, photosensitizer, and oxygen to create activated singlet 
oxygen $\left({ }^{1} \mathrm{O}_{2}\right)$. to kill cells. To address the need for better dosimetry in PDT, we have developed a singlet oxygen explicit dosimetry model to predict PDT outcome ${ }^{5-8}$. The four major photochemical parameters in a macroscopic singlet oxygen model have been investigated and determined for the photosensitizer benzoporphyrin derivative monoacid A (BPD).

In this study, reactive oxygen species explicit dosimetry (ROSED) was performed using measured values of light fluence rate, photosensitizer concentration and [ROS] to evaluate the treatment outcomes of BPD-mediated vascular PDT in mice bearing radiation-induced fibrosarcoma (RIF) tumors. Vascular-targeted PDT can be achieved using a short (15 minute) drug light interval ${ }^{9}$. By inducing vascular shutdown, nutrient supply and removal of metabolic waste is halted which results in RIF tumor cell death. This is beneficial because tumor vasculature is easy to access for targeting, and vascular damage is efficient in cancer cell killing and has a low likelihood of leading to drug resistance.

\section{MATERIALS AND METHODS}

\section{Tumor Model}

RIF cells were cultured and $30 \mu \mathrm{l}$ were injected at $1 \times 10^{7}$ cells $/ \mathrm{ml}$ intradermally over the right shoulders of 6 to 8 weeks old female $\mathrm{C} 3 \mathrm{H}$ mice (NCI-Frederick, Frederick, Maryland), as described previously ${ }^{10-12}$. Animals were under the care of the University of Pennsylvania Laboratory Animal Resources. All studies were approved by the University of Pennsylvania Institutional Animal Care and Use Committee. Tumors were treated at a size of $\sim 3$ to $5 \mathrm{~mm}$ in diameter. Fur within the region of tumor inoculation was clipped prior to injection of cells, and the treatment area was depilated with Nair (Church \& Dwight Co., Inc., Ewing, New Jersey) at least $24 \mathrm{~h}$ before measurements. Mice were provided a chlorophyll-free (alfalfafree) rodent diet (Harlan Laboratories Inc., Indianapolis, Indiana) starting at least 10 days prior to treatment to eliminate the fluorescence signal from chlorophyll-breakdown products, which have a similar emission range to the BPD fluorescence spectra used to determine the concentration of BPD in the tumor. During the delivery of PDT, mice were kept under anesthesia on a heat pad at $38^{\circ} \mathrm{C}$.

\section{PDT Treatment Conditions}

PDT was delivered using an optical fiber with a microlens attachment coupled to a diode laser. A $690 \mathrm{~nm}$ laser (B\&W Tek Inc., Newark, Delaware) was used for PDT after a $15 \mathrm{~min}$ drug-light interval. The in-air fluence rate $\left(\phi_{\text {air }}\right)$ is defined as the calculated irradiance determined by the laser power divided by the treatment area $(1 \mathrm{~cm}$ diameter spot size $)$. The inair fluence was calculated by multiplying the in-air fluence rate by the treatment time. RIF tumor-bearing mice with no photosensitizer and no light excitation were used as controls (n =5). Treatment conditions are summarized in Table 1 .

BPD (trademark Visudyne ${ }^{\circledR}$ ) at a dosage of $1 \mathrm{mg} / \mathrm{kg}$ was injected through the mouse tail vein as described previously. ${ }^{12,13}$ At a 15 min drug-light interval, superficial irradiation of the tumor was performed with a 690-nm laser (B\&W Tek Inc., Newark, Delaware). A microlens fiber was coupled to the laser to irradiate the tumor uniformly. Animals were 
assigned to four light dose groups, and each group was comprised of 2 to 3 subgroups with different $\phi$. There were a total of 6 treatment groups: $22.5 \mathrm{~J} / \mathrm{cm}^{2}$ at $75 \mathrm{Mw} / \mathrm{cm}^{2}, 30 \mathrm{~J} / \mathrm{cm}^{2}$ at $75 \mathrm{~mW} / \mathrm{cm}^{2}, 45 \mathrm{~mW} / \mathrm{cm}^{2}$ at $75 \mathrm{~mW} / \mathrm{cm}^{2}, 50 \mathrm{~J} / \mathrm{cm}^{2}$ at $75 \mathrm{~mW} / \mathrm{cm}^{2}, 70 \mathrm{~J} / \mathrm{cm}^{2}$ at $150 \mathrm{~mW} / \mathrm{cm}^{2}$ and $100 \mathrm{~J} / \mathrm{cm}^{2}$ at $150 \mathrm{~mW} / \mathrm{cm}^{2}$. Tumor-bearing mice that received neither light irradiation nor BPD were used as controls.

\section{Oxygen Measurements}

The in vivo tissue oxygen partial pressure $\mathrm{pO}_{2}$ was measured during PDT treatment using a phosphorescence-based ROS probe (OxyLite Pro, Oxford Optronix, Oxford, United Kingdom). A bare-fiber-type probe (NX-BF/O/E, Oxford Optronix, Oxford, United Kingdom) was placed inside the tumor at a $3 \mathrm{~mm}$ depth from the treatment surface. The ROS concentration ([ROS]) was calculated by multiplying the measured $\mathrm{pO}_{2}$ with the ROS solubility in tissue, which is $1.295 \mu \mathrm{M} / \mathrm{mmHg} .{ }^{5}$ Measured [ROS $]_{0}$ and [ROS](t) were used to calculate reacted oxygen species using the macroscopic singlet oxygen model. ${ }^{14,15}$

\section{BPD Concentration}

Following the drug-light interval of 15 min, measurements of light fluence rate, photosensitizer concentration and [ROS] were performed. BPD fluorescence spectra was obtained using a custom-made multi-fiber contact probe before and after PDT. ${ }^{17}$ The probe is connected to a $405 \mathrm{~nm}$ laser (Power Technology Inc., Little Rock, AR, USA) for the fluorescence excitation of BPD and a multichannel CCD spectrograph (InSpectrum, Princeton Instruments, Trenton, NJ, USA) for the collection of the spectra. The in vivo photosensitizer concentration was obtained by comparing the in vivo BPD spectra with those of phantoms with known photosensitizer concentrations. The attenuation of the fluorescence signal due to light absorption and scattering by tissues was corrected by applying an empirical correction factor described elsewhere. ${ }^{16}$ The accuracy of in vivo measurements was validated by ex vivo measurements in separate mice. ${ }^{19}$

\section{Tumor Regrowth Rate Analysis}

Tumor volumes were measured daily after PDT . Width (a) and length $(b)$ were measured with slide calibers, and tumor volumes ( $V$ ) was calculated using $V=\pi \times a^{2} \times b / 6{ }^{20}$ Tumor volumes were tracked for 14 days, and the tumor regrowth factor $(k)$ was calculated by the best exponential fit [with a form $f(d)=\mathrm{Ae}^{k d}$ ] to the measured volumes over the days (d). CI was calculated for each treatment group as

$$
C I=1-\frac{k}{k_{c t r}}
$$

where $k$ is the tumor regrowth factor for each group and $k_{\mathrm{ctr}}$ is the regrowth factor for the control group, which consisted of tumors exposed to neither BPD nor light illumination. 


\section{Reactive Oxygen Species Explicit Dosimetry}

Type II PDT process can be described by a set of kinetic equations which can be simplified to describe the creation of $[R O S]_{\mathrm{rx}} \cdot{ }^{21,22}$ These equations are dependent on the temporal and spatial distribution of $\phi$, photosensitizer concentration $\left(\left[\mathrm{S}_{0}\right]\right)$, ground state oxygen concentration ([ROS]), and the photosensitizer-specific reaction-rate parameters ( $\beta$ and $\xi)$. The relevant equations are:

$$
[R O S]_{r x}=\int_{t=0}^{T} \xi \frac{\left[{ }^{3} O_{2}\right]}{\left[{ }^{3} O_{2}\right]+\beta} \phi\left[S_{0}\right] \mathrm{dt}
$$

where $\phi$ is the light fluence rate, $S$ is the source term, $\mu_{a}$ and $\mu_{s}$ ' are the absorption and reduced scattering coefficients, respectively. The five parameters involved in the kinetic equations are photosensitizer-specific and details of each can be found elsewhere. ${ }^{21} \xi$ is the photochemical oxygen consumption rate per light fluence rate and photosensitizer concentration under ample ROS supply. $\beta$ represents the ratio of the monomolecular decay rate of the triplet state photosensitizer to the bimolecular rate of the triplet photosensitizer quenching by ROS. $g$ is the maximum macroscopic oxygen perfusion rate. The reacted oxygen species concentration ([ROS $]_{\mathrm{rx}}$ ) used as a dosimetric measure in other studies was calculated and compared using an initial ground state oxygen concentration of ([ROS $]_{0}$ ) of $40 \mu \mathrm{M}$, the measured [ROS $]_{0}$, the measured [ROS $]_{(\mathrm{t})}$ throughout treatment, and the [ROS] determined with blood flow changes measured during treatment. The two parameters used in Eq. (2) are obtained from literature: $\zeta=(55 \pm 15) \times 10^{-3} \mathrm{~cm}^{2} \mathrm{~s}^{-1} \mathrm{~mW}^{-1}$ and $\beta=11.9 \mu \mathrm{M}$. $^{1}$

\section{RESULTS AND DISCUSSION}

BPD-mediated PDT with different in-air fluences, different $\phi_{a i i}$, and different exposure times was performed in mouse models bearing RIF tumors. Tissue optical properties, photosensitizer concentration, and tissue oxygenation were measured to calculate PDT dose, and $[R O S]_{\mathrm{rx}}$. Table 1 summarizes all of the treatment conditions, BPD concentration was measured both before and after PDT treatment. It is shown in Figure 1, the symbols represent the measured values, and the solid lines are model-calculated photosensitizer concentration during treatment. There are not significant changed.

Measured $\left[{ }^{3} \mathrm{O}_{2}\right]$ was used to refine the photochemical parameters previously determined for the reactive oxygen species explicit dosimetry model used to calculate $[\mathrm{ROS}]_{\mathrm{rx}}$, Individually, measured $\left[{ }^{3} \mathrm{O}_{2}\right]_{(\mathrm{t})}$ for each mouse was used as shown Figure 2. There are no significant changes during the treatment.

Fluence, PDT dose and calculated $[\mathrm{ROS}]_{\mathrm{rx}}$ at $3 \mathrm{~mm}$ were compared as dosimetric quantities to estimate the outcome of BPD-mediated PDT for RIF tumors. Outcome was quantified by the calculation of CI. If there is no tumor regrowth up to 14 days after treatment, then $\mathrm{CI}=$ 1. PDT dose is calculated using the product of PS uptake and measured light fluence rate at $3 \mathrm{~mm}$. The fluence rate at $3 \mathrm{~mm}$ is determined. We used Eq. (2) ${ }^{19}$ and photochemical 
parameters $\left(\zeta\right.$ and $\beta$ ) obtained from literature ${ }^{13}$ to calculate $[R O S]_{\mathrm{rx}}$. The goodness of the fit and the corresponding upper and lower bounds of the fits (gray area) to the fluence, PDT dose and the calculated $[R O S]_{\mathrm{rx}}$ are presented in Figure 3. Figure 3(a) shows that, while fluence correlates sigmoidal with the PDT outcome, it exhibits large uncertainties as defined by the large bounds of the gray area, as well as by the low value of $\mathrm{R}^{2}=0.6296$. As shown in Figure 3(b), PDT dose allows for reduced subject variation and improved predictive efficacy as compared to fluence and measured [ROS $]_{\mathrm{rx}}$. PDT dose showed a better correlation with CI with a higher value of $\mathrm{R}^{2}=0.918$ and a narrower band of gray area as it accounts for both light dose and tissue [BPD] levels. However, PDT dose overestimates $[R O S]_{\mathrm{rx}}$ in the presence of hypoxia as it does not account for the oxygen dependence of ROS quantum yield. The goodness of fit $R^{2}=0.9843$ and the narrowest gray area in Figure 3 (c) shows that the measured $[R O S]_{\mathrm{rx}}$ correlates the best with CI. $[\mathrm{ROS}]_{\mathrm{rx}}$ accounts for the key quantities of light fluence, photosensitizer concentration, and tissue oxygen level.

\section{CONCLUSION}

The response of mouse RIF tumors to PDT depends on tissue oxygenation, photosensitizer uptake, total energy delivered, and the $\phi$ at which treatment is delivered. An accurate dosimetry quantity for the evaluation of treatment outcome should account for all of these parameters. This study evaluated the efficacy and outcomes of different PDT treatments and how fluence, PDT dose, and $[\mathrm{ROS}]_{\mathrm{rx}}$ compare as dosimetric quantities. The correlation between $\mathrm{CI}$ and $[R O S]_{\mathrm{rx}}$ suggests that $[\mathrm{ROS}]_{\mathrm{rx}}$ at $3 \mathrm{~mm}$ is the best quantity to predict the treatment outcome for a clinically relevant tumor regrowth endpoint. PDT dose is a better dosimetric quantity than fluence, but it is worse than $[R O S]_{\mathrm{rx}}$ as it does not account for the consumption of $\left[{ }^{3} \mathrm{O}_{2}\right]$ for different $\phi$. For BPD in RIF tumors, the temporal dependence of in-vivo oxygen concentration during PDT can't be well modeled by our macroscopic model (for measurement [ROS], $\mathrm{R}^{2}=0.9843$ ). This implies that it is necessary to make ROSED measurements during PDT to determine $[\mathrm{ROS}]_{\mathrm{rx}}$, in conjunction with the photochemical parameters required in Eq.2. This study determines the $[\mathrm{ROS}]_{\mathrm{rx}}$ threshold dose for vascular BPD-mediated PDT for the first time.

\section{Acknowledgments}

The authors would like to thank Min Yuan, Joann Miller and Shirron Carter for their advice concerning the mouse studies and protocols. This work was supported by grants from the National Institute of Health (NIH) R44 CA183236, R01 CA85831 and P01 CA87971.

\section{REFERENCES}

[1]. Agostinis P, Berg K, Cengel KA, et al., "Photodynamic therapy of cancer: an update," CA: a cancer journal for clinicians 61(4), 250-81 (2011). [PubMed: 21617154]

[2]. Castano AP, Demidova TN, Hamblin MR, "Mechanisms in photodynamic therapy: part onephotosensitizers, photochemistry and cellular localization," Photodiagnosis and photodynamic therapy 1(4), 279-93 (2004). [PubMed: 25048432]

[3]. Penjweini R, Kim MM, Liu B, Zhu TC, "Evaluation of the 2-(1-Hexyloxyethyl)-2-devinyl pyropheophorbide(HPPH) mediated photodynamic therapy by macroscopic singlet oxygen modeling,” Journal of Biophotonics 9(11-12),1344-1354 (2016). [PubMed: 27653233] 
[4]. Qiu H, Kim MM, Penjweini R, Zhu TC, "Macroscopic singlet oxygen modeling for dosimetry of Photofrinmediated photodynamic therapy: an in-vivo study," Journal of biomedical optics 21(8), 88002 (2016). [PubMed: 27552311]

[5]. Wang KKH, Finay JC, Busch TM et al., "Explicit dosimetry for photodynamic therapy: macroscopic singlet oxygen modeling," Journal of Biophotonics, 3(5-6), 304-318 (2010). [PubMed: 20222102]

[6]. Liang X, Wang KKH, Zhu TC, "Singlet oxygen dosimetry modeling for photodynamic therapy," Proc. SPIE, 8210, 8210T-1-8210T-7 (2012).

[7]. Mcillan DD, Chen D, Kim MM, Liang X, Zhu TC, "Parameter determination for singlet oxygen modeling of BPD-mediated PDT," Proc. SPIE, 8568, 856810-1-856810-8 (2013).

[8]. Zhu TC, Finlay JC, Zhou X, Li J, "Macroscopic modeling of the singlet oxygen production during PDT,” Proc. SPIE, 6427, 642708-1-642708-12 (2007).

[9]. Chen B, Pogue BW, Luna JM, Hardman RL, Hoopes PJ, Hasan T, “Tumor Vascular Permeabilization by Vascular-Targeting Photosensitization: Effects, Mechanism, and Therapeutic Implications," Clin. Cancer Res, 12(1), 917-23 (2006). [PubMed: 16467106]

[10]. Wang KK et al., "Explicit dosimetry for photodynamic therapy: macroscopic singlet oxygen modeling," J. Biophoton. 3(5-6), 304-318 (2010).

[11]. Zhu TC et al., "In-vivo singlet oxygen threshold doses for PDT," Photon. Lasers Med. 4(1), 59$71(2015)$.

[12]. Liu B et al., "Comparison of PDT parameters for RIF and H460 tumor models during HPPHmediated PDT,” Proc. SPIE $893189311 \mathrm{C}$ (2014).

[13]. Kim MM. Ghogare AA, Greer A and Zhu TC, "On the in vivo photochemical rate parameters for PDT reactive oxygen species modeling". Phys. Med. Biol. 00(2016) 1-48.

[14]. Qiu H, Kim MM, Penjweini R, Zhu TC, "Dosimetry study of PHOTOFRIN-mediated photodynamic therapy in a mouse tumor model," Proc. SPIE 9694, 96940T (2016).

[15]. Penjweini R, Liu B, Kim MM, Zhu TC, "Explicit dosimetry for 2-(1-hexyloxyethyl)-2-devinyl pyropheophorbide-a-mediated photodynamic therapy: macroscopic singlet oxygen modeling," Journal of biomedical optics 20(12), 128003 (2015).

[16]. Mesquita RC et al., "Tumor blood flow differs between mouse strains: consequences for vasoresponse to photodynamic therapy," PLoS One 7(5), e37322 (2012).

[17]. Penjweini R, Kim MM, Liu B, and Zhu TC, "Evaluation of the 2-(1-Hexyloxyethyl)-2devinylpyropheophorbide (HPPH) mediated photodynamic therapy by macroscopic singlet oxygen modeling,” J. Biophotonics 9(11-12): 1344-1354 (2016). [PubMed: 27653233]

[18]. Qiu H, Kim MM, Penjweini R, and Zhu TC, "Macroscopic singlet oxygen modeling for dosimetry of Photofrinmediated photodynamic therapy: an in vivo study," J. Biomed. Opt. 21(8): 088002 (2016).

[19]. Kim MM, Penjweini R, and Zhu TC, "Evaluation of singlet oxygen explicit dosimetry (SOED) for predicting treatment outcomes of benzoporphyrin derivative monoacid ring A (BPD-MA)mediated photodynamic therapy," J. Biomed. Opt. 22(2): 028002 (2017).

[20]. Busch TM et al., "Fluence rate-dependent intratumor heterogeneity in physiologic and cytotoxic responses to photofrin photodynamic therapy," Photochem. Photobiol. Sci. 8(12), 1683-1693 (2009). [PubMed: 20024165]

[21]. Kim MM, Penjweini R, Ong YH and Zhu TC, "Singlet oxygen explicit dosimetry to predict longterm local tumor control for BPD-mediated photodynamic therapy," Proc. SPIE 10047, 100470X(2018).

[22]. Hu XH, Feng Y, Lu JQ, et al., "Modeling of a type II photofrin-mediated photodynamic therapy process in a heterogeneous tissue phantom," Photochemistry and photobiology 81(6), 1460-8 (2005). [PubMed: 15960591] 


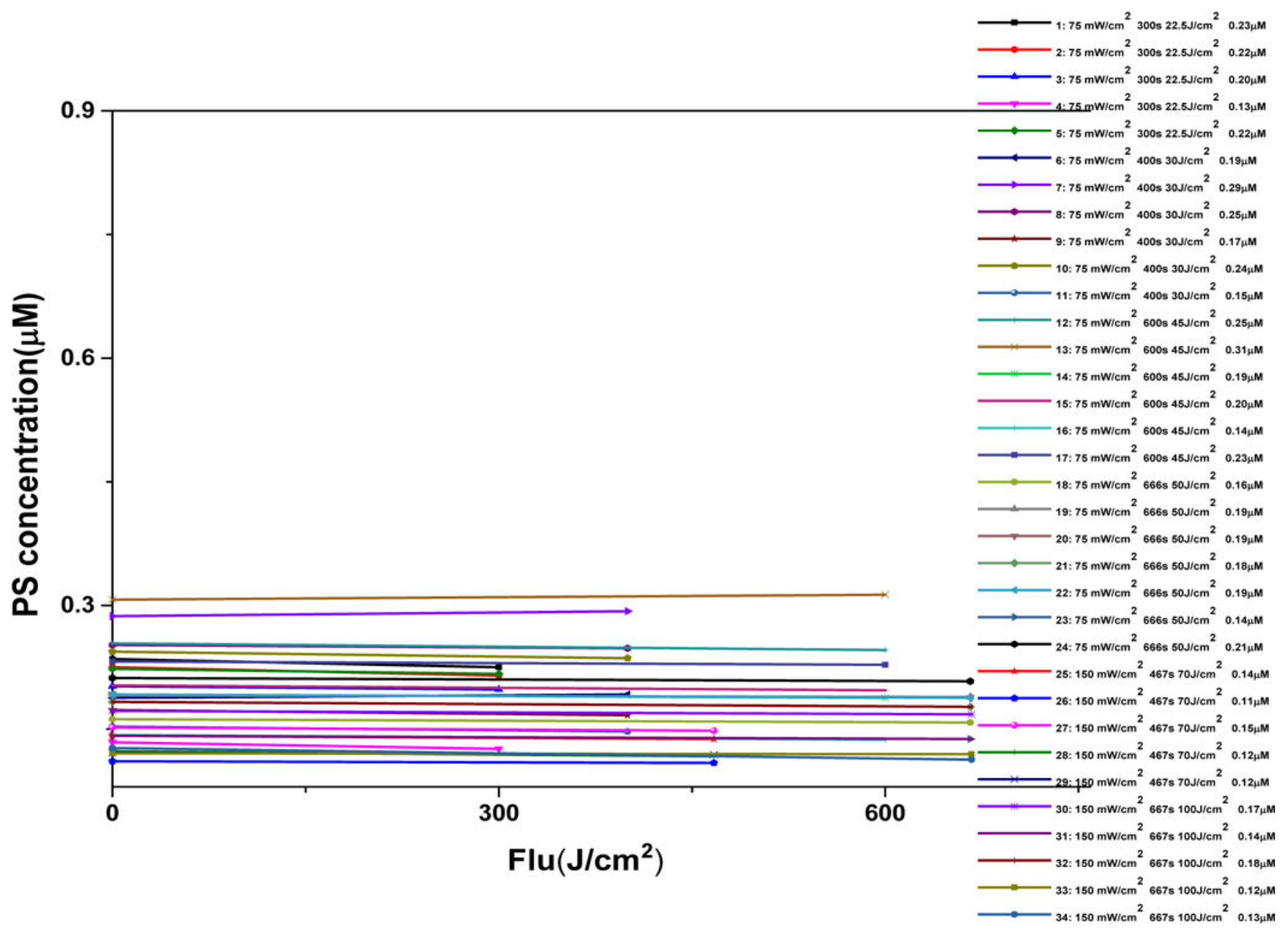

Figure 1.

BPD concentration versus fluence at $3 \mathrm{~mm}$ tumor depth for various treatment conditions.

The lines indicate the calculated change in photosensitizer concentration during light delivery for PDT. 


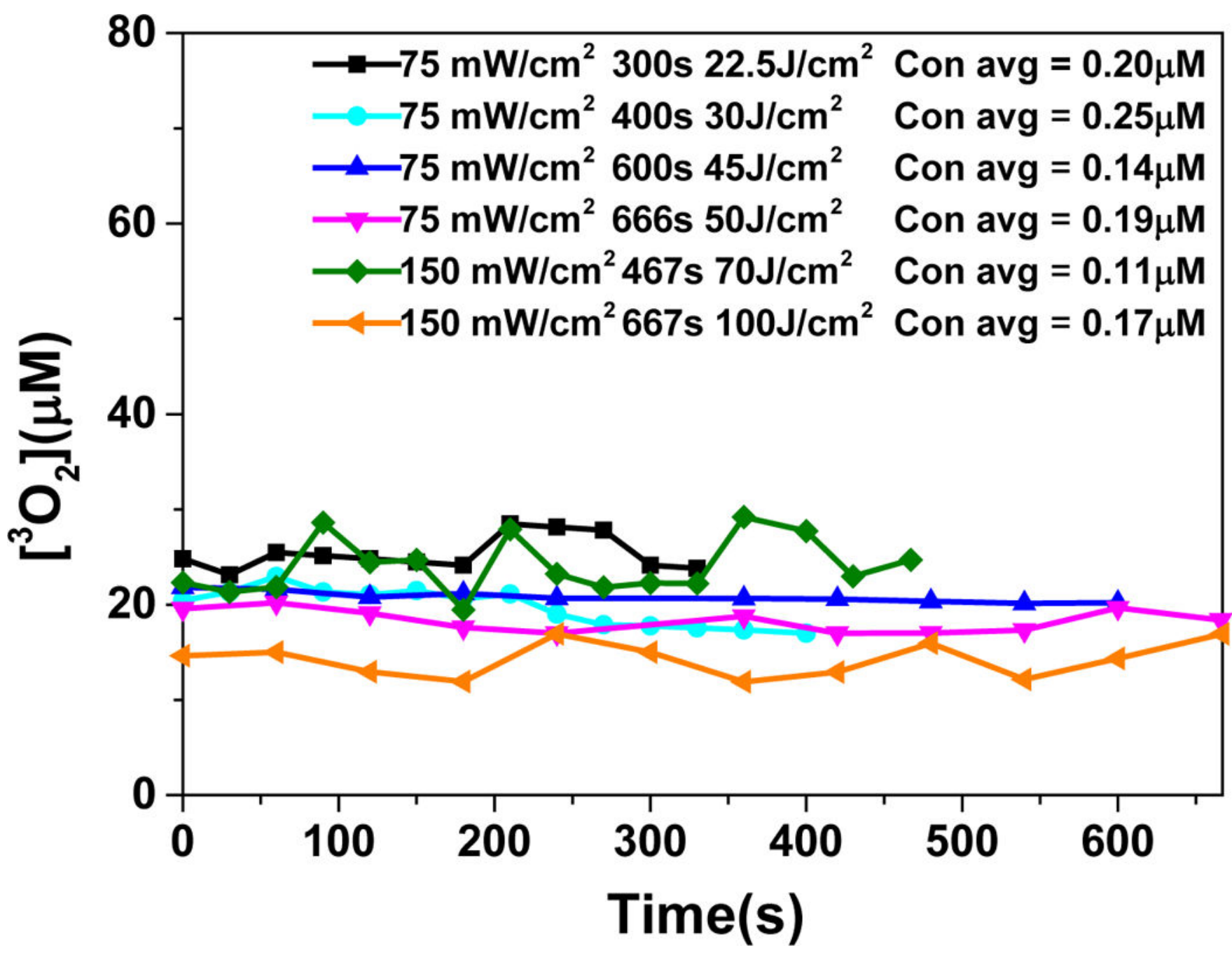

Figure 2.

Temporal changes in ROS concentration during light for PDT with various treatment conditions. The lines represent the calculated changed in ROS concentration during treatment. 
(a)

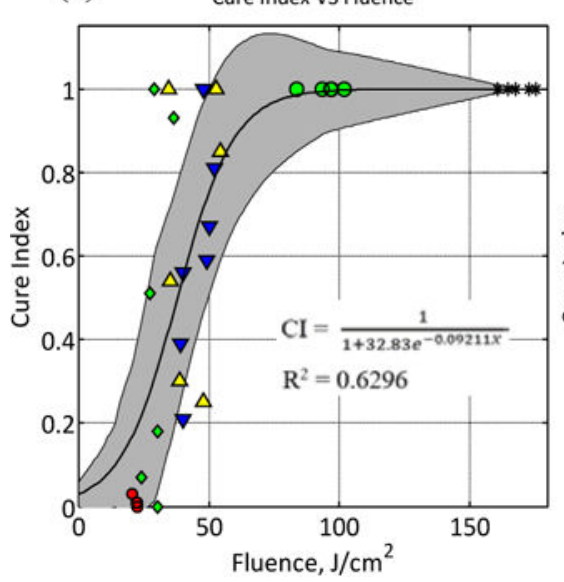

(b)

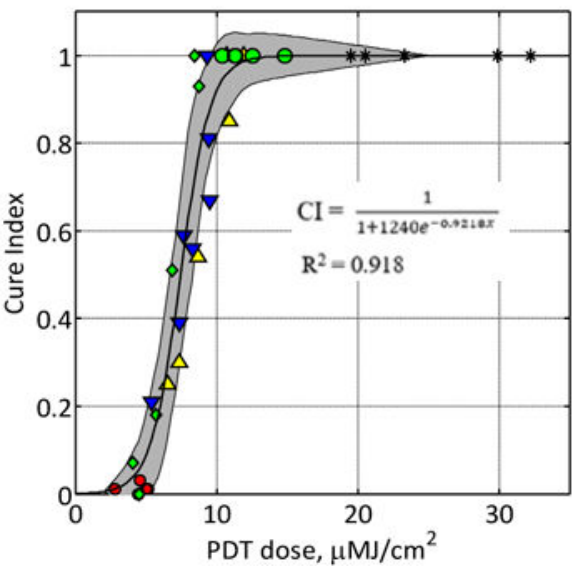

(c)

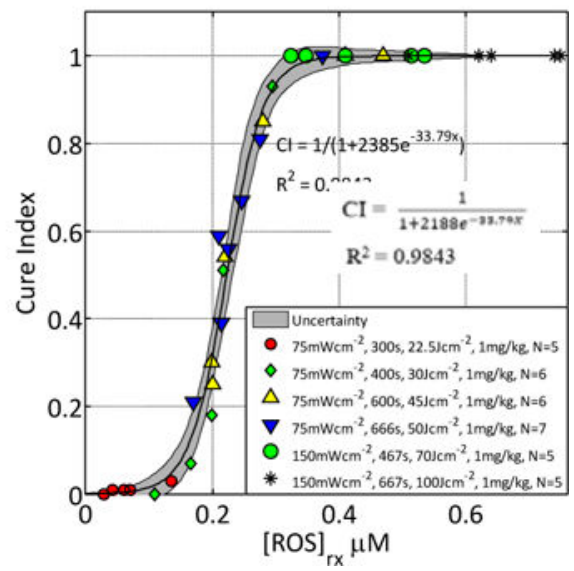

Figure 3.

CI plotted as a function of (a) fluence at a $3 \mathrm{~mm}$ tumor depth, (b) calculated PDT dose at 3 $\mathrm{mm}$ depth, and (c) mean reacted oxygen species at $3 \mathrm{~mm}$ depth ([ROS $]_{\mathrm{rx}}$ ) calculated using Eq. (2) and the parameters summarized are $\zeta=(55 \pm 15) \times 10^{-3} \mathrm{~cm}^{2} \mathrm{~s}^{-1} \mathrm{~mW}^{-1}$ and $\beta=11.9 \mu \mathrm{M}$. The solid lines show the best-fit to the data with functional forms $\mathrm{CI}=\frac{1}{1+32.83 e^{-0.09211 x}}, \mathrm{CI}=\frac{1}{1+1240 e^{-0.9218 x}}$, and $\frac{1}{1+2188 e^{-33.79 x}}$ with $\mathrm{R}^{2}=0.6296$, 0.918 and 0.9843 for (a), (b), respectively. The gray region indicates the upper and lower bounds of the fit with $95 \%$ confidence level. 
Table 1:

In-air light fluence, in-air light fluence rate, BPD concentration in tumors, initial tissue oxygenation, PDT dose, as well as calculated reactive oxygen species concentration.

\begin{tabular}{|c|c|c|c|c|c|c|c|c|c|c|}
\hline Index & Mice \# & $\begin{array}{c}\text { Fluence } \\
\text { rate in air } \\
\text { (expected) } \\
\mathbf{m W} / \mathrm{cm}^{2}\end{array}$ & $\begin{array}{c}\text { Fluenc } \\
\text { e rate } \\
\text { (measu } \\
\text { red) on } \\
\text { surface } \\
\mathrm{mW} / \mathrm{c} \\
\mathbf{m}^{2}\end{array}$ & $\begin{array}{c}\text { Fluence } \\
\text { rate in } \\
\text { tissue } \\
(3 \mathrm{~mm}) \\
\mathrm{mW} / \mathrm{cm}^{2}\end{array}$ & $\begin{array}{c}\text { Total } \\
\text { treatment } \\
\text { time } \\
\text { seconds }\end{array}$ & $\begin{array}{c}\text { BPD } \\
\text { concentration } \\
\mu \mathrm{M}\end{array}$ & $\begin{array}{c}\text { PDT } \\
\text { dose } \\
\mu \mathrm{MJ} / \mathrm{cm}^{2}\end{array}$ & $\begin{array}{c}{[\mathbf{R O S}]} \\
\mathbf{R x} \\
\boldsymbol{\mu M}\end{array}$ & $\mathbf{k}$ & CI \\
\hline 1 & \#15-4 & 75.00 & 95.70 & 73.62 & 300.00 & 0.23 & 5.10 & 0.07 & 0.41 & 0.01 \\
\hline 2 & \#15-5 & 75.00 & 96.90 & 74.54 & 300.00 & 0.22 & 4.97 & 0.06 & 0.41 & 0.01 \\
\hline 3 & \#14-3 & 75.00 & 97.20 & 74.77 & 300.00 & 0.20 & 4.43 & 0.03 & 0.42 & 0.00 \\
\hline 4 & \#16-4 & 75.00 & 95.00 & 73.08 & 300.00 & 0.13 & 2.77 & 0.04 & 0.41 & 0.01 \\
\hline 5 & \#16-5 & 75.00 & 89.00 & 68.46 & 300.00 & 0.22 & 4.58 & 0.14 & 0.40 & 0.03 \\
\hline 6 & $\# 8-1$ & 75.00 & 98.00 & 75.38 & 400.00 & 0.19 & 5.70 & 0.20 & 0.34 & 0.18 \\
\hline 7 & $\# 8-2$ & 75.00 & 88.00 & 67.69 & 400.00 & 0.29 & 7.85 & 0.32 & 0.00 & 1.00 \\
\hline 8 & $\# 8-3$ & 75.00 & 87.00 & 68.15 & 400.00 & 0.25 & 6.82 & 0.22 & 0.20 & 0.51 \\
\hline 9 & \#17-5 & 75.00 & 78.00 & 60.00 & 400.00 & 0.17 & 4.08 & 0.17 & 0.38 & 0.07 \\
\hline 10 & \#18-1 & 75.00 & 118.00 & 90.77 & 400.00 & 0.24 & 8.71 & 0.29 & 0.03 & 0.93 \\
\hline 11 & \#18-2 & 75.00 & 98.00 & 75.38 & 400.00 & 0.15 & 4.52 & 0.11 & 0.42 & 0.00 \\
\hline 12 & \#8-4 & 75.00 & 76.00 & 58.46 & 600.00 & 0.25 & 8.70 & 0.22 & 0.19 & 0.54 \\
\hline 13 & $\# 8-5$ & 75.00 & 75.00 & 57.69 & 600.00 & 0.31 & 10.69 & 0.41 & 0.00 & 1.00 \\
\hline 14 & \#9-1 & 75.00 & 84.00 & 64.62 & 600.00 & 0.19 & 7.37 & 0.20 & 0.29 & 0.30 \\
\hline 15 & \#15-1 & 75.00 & 118.00 & 90.77 & 600.00 & 0.20 & 10.91 & 0.28 & 0.06 & 0.85 \\
\hline 16 & $\# 15-2$ & 75.00 & 104.00 & 80.00 & 600.00 & 0.14 & 6.54 & 0.20 & 0.31 & 0.25 \\
\hline 17 & \#15-3 & 75.00 & 114.00 & 87.69 & 600.00 & 0.23 & 11.91 & 0.47 & 0.00 & 1.00 \\
\hline 18 & \#16-1 & 75.00 & 96.00 & 73.85 & 666.00 & 0.16 & 7.66 & 0.21 & 0.17 & 0.59 \\
\hline 19 & \#16-2 & 75.00 & 98.00 & 75.38 & 666.00 & 0.19 & 9.50 & 0.25 & 0.14 & 0.67 \\
\hline 20 & \#16-3 & 75.00 & 94.00 & 72.31 & 666.00 & 0.19 & 9.28 & 0.37 & 0.00 & 1.00 \\
\hline 21 & \#17-1 & 75.00 & 102.00 & 78.46 & 666.00 & 0.18 & 9.42 & 0.28 & 0.08 & 0.81 \\
\hline 22 & $\# 17-2$ & 75.00 & 76.00 & 58.46 & 666.00 & 0.19 & 7.37 & 0.21 & 0.25 & 0.39 \\
\hline 23 & \#17-3 & 75.00 & 78.00 & 60.00 & 666.00 & 0.14 & 5.40 & 0.17 & 0.33 & 0.21 \\
\hline 24 & \#17-4 & 75.00 & 78.00 & 60.00 & 666.00 & 0.21 & 8.30 & 0.23 & 0.18 & 0.56 \\
\hline 25 & $\# 3-2$ & 150.00 & 233.00 & 179.23 & 467.00 & 0.14 & 11.30 & 0.35 & 0.00 & 1.00 \\
\hline 26 & $\# 3-3$ & 150.00 & 260.00 & 200.00 & 467.00 & 0.11 & 10.38 & 0.32 & 0.00 & 1.00 \\
\hline 27 & $\# 3-4$ & 150.00 & 269.00 & 206.92 & 467.00 & 0.15 & 14.78 & 0.53 & 0.00 & 1.00 \\
\hline 28 & $\# 3-5$ & 150.00 & 284.00 & 218.46 & 467.00 & 0.12 & 12.53 & 0.51 & 0.00 & 1.00 \\
\hline 29 & \#A-3 & 150.00 & 270.00 & 207.69 & 467.00 & 0.12 & 11.29 & 0.41 & 0.00 & 1.00 \\
\hline 30 & \#4-1 & 150.0 & 268.00 & 259.00 & 667.00 & 0.17 & 29.86 & 0.75 & 0.00 & 1.00 \\
\hline 31 & $\# 4-2$ & 150.0 & 255.00 & 246.40 & 667.00 & 0.14 & 23.32 & 0.51 & 0.00 & 1.00 \\
\hline 32 & \#4-3 & 150.0 & 272.00 & 262.80 & 667.00 & 0.18 & 32.18 & 0.74 & 0.00 & 1.00 \\
\hline 33 & $\# 4-4$ & 150.0 & 260.00 & 251.00 & 667.00 & 0.12 & 19.51 & 0.62 & 0.00 & 1.00 \\
\hline 34 & \#A-4 & 150.0 & 249.00 & 240.60 & 667.00 & 0.13 & 20.53 & 0.64 & 0.00 & 1.00 \\
\hline
\end{tabular}

Proc SPIE Int Soc Opt Eng. Author manuscript; available in PMC 2019 May 10. 